\title{
Molecules and functions of Cornus officinalis bark volatiles
}

\author{
Xiaochen Yue ${ }^{1}$, Ximei Li ${ }^{1}$, Xiangmeng Chen ${ }^{2}$, Muhammad Aqeel Ashraf ${ }^{3}$, Zhenling Liư ${ }^{4}$, Huitao Bi ${ }^{1}$, \\ Dongfang Zheng', Yong Zhao', Wanxi Peng ${ }^{1 *}$
}

${ }^{1}$ School of Forestry, Henan Agricultural University, Zhengzhou 450002, China, ${ }^{2}$ School of Chemical Engineering and Energy, Zhengzhou University, Zhengzhou 450001, China, ${ }^{3}$ Department of Geology Faculty of Science, University of Malaya, 50603 Kuala Lumpur, Malaysia, ${ }^{4}$ School of Management, Henan University of Technology, Zhengzhou 450001, China

\section{A B S T R A C T}

\begin{abstract}
Cornus officinalis Sieb. et Zucc is a traditional Chinese valuable medicinal material. Clinically, it is customary to use ripe fruits from which seeds have been removed for medicinal purposes. The pulp contains 16 amino acids and a large number of essential elements for the human body. In recent years, with the expansion of the application of cornus officinalis, its pharmacological and pharmacological effects have been increasingly studied. At present, significant achievements have been made in the study of the bioactive components of cornus officinalis. The research of these achievements has been based on the research of the fruit of cornus officinalis. The study of branches or bark of cornus officinalis is very rare. With the fruit of cornus officinalis getting more and more attention, in order to solve the problem of the shortage of cornus officinalis fruit in the market, in this paper, starting from the study of bark of cornus officinalis, TGA-DTG and PY-GC-MS analysis methods were used to study the weight loss and pyrolysis of cornus officinalis bark, providing a basis for more fully utilizing cornus officinalis resources. With reference.
\end{abstract}

Keywords: Cornus officinalis bark; Volatiles; Value utilization; PY-GC-MS; TGA-DTG

\section{INTRODUCTION}

Cornus officinalis Sieb. et Zucc, the original plant is a deciduous tree or shrub, which is mainly distributed in the north of the Yangtze River in China, in the middle and lower hills of the Qinling Mountains, the south of the Funiu Mountain and the Tianmu Mountain in Zhejiang, and its main planting province is Zhejiang, Henan, Shandong, Anhui and other places (Zhao, 1992; Hong, 2003; Han, 2005). Cornus officinalis contains various chemical components such as volatile oils, organic acids and iridoid glycosides, and has a variety of pharmacological activities (Khan, 2018; Hasan, 2018; Howlader et al., 2018). According to traditional Chinese medicine, cornus officinalis has the effect of tonifying liver and kidney, astringent essence, and modern pharmacology research shows that cornus officinalis has various clinical features such as hypoglycemic, anti-bleeding, platelet aggregation inhibition, anti-inflammatory, anti-oxidation, antibacterial and anti-tumor effects (Ren, 2016; Huang et al., 2017; He et al., 2016; Miyazawa et al., 2014; Cao and Lei, 2013). Efficacy. In recent years, the study of its chemical composition has also been carried out in depth (El Toum, 2018; Khan et al., 2018). Studies have shown that in the decontamination study of Bawei Pills, only cornus officinalis has hypoglycemic effect on streptozotocininduced rat model of diabetes (Ma et al., 2014; Qian et al., 2001; Chen et al., 2016) cornus officinalis can kill ascites cancer cells in vitro. Clinically used for radiotherapy, chemotherapy, leukopenia, primary liver cancer, metastatic liver cancer (Telang et al., 2016; Miyazawa and Kameoka, 2014; Telang et al., 2012), etc.; In addition, ethanol extract cornus officinalis can significantly reduce normal blood glucose, serum total cholesterol and triacylglycerol; and cornus officinalis Oleanolic acid has a slight cardiac diuretic effect and is also clinically used to treat acute viral hepatitis (Hsu et al., 2006; Wang et al., 2007; Jang et al., 2014).

In this paper, the bark of cornus officinalis as the research object, the bark was dried and made into powder, and analyzed by TGA-DTG and PY-GC-MS. It provides scientific research on the utilization of cornus officinalis from a new perspective and direction in accordance with. 


\section{MATERIAL AND METHODS}

\section{Experimental materials}

Cornus officinalis bark was obtained from Nanyang City, Henan Province, Xixia County Forest Farm. Place the bark in a $100^{\circ} \mathrm{C}$ oven until absolutely dry. Then use a pulverizer to break the bark as a sample to be tested.

\section{Experimental methods TGA-DTG analysis}

The sample of the fruit of Cornus officinalis were analyzed viathermogravimetric analyzer (TGA Q50 V20. 8 Build 34). The nitrogen release rate was $60 \mathrm{ml} / \mathrm{min}$. The temperature program of TG started at room temperature and increased to $300^{\circ} \mathrm{C}$ at a rate of $5^{\circ} \mathrm{C} / \mathrm{min}$ (Kok et al., 2016; Gornicka and Gorecki, 2010; Trivedi et al., 2017).

PY-GC-MS Analysis The fruit of cornus officinalis was analyzed viathermal cracking-gas chromatography-mass spectrometry (CDS5000-Agilent7890B-5977A). The carrier gas was high purity helium, the pyrolysis temperature was $500^{\circ} \mathrm{C}$, the heating rate was $20^{\circ} \mathrm{C} / \mathrm{ms}$, and the pyrolysis time was $15 \mathrm{~s}$. The pyrolysis product transfer line and the injection valve temperature were set to $300^{\circ} \mathrm{C}$; Column HP-5MS; Capillary column $(30 \mathrm{~m} \times 0.25 \mathrm{~mm} \times 0.25 \mu \mathrm{m})$; Shunt mode, split ratio of 1:60, shunt rate of $50 \mathrm{~mL} / \mathrm{min}$. The temperature of the GC program started at $40^{\circ} \mathrm{C}$ for $2 \mathrm{~min}$, increased to $120^{\circ} \mathrm{C}$ at a rate of $5^{\circ} \mathrm{C} / \mathrm{min}$, and then increased to $200^{\circ} \mathrm{C}$ at a rate of $10^{\circ} \mathrm{C} / \mathrm{min}$ for $15 \mathrm{~min}$. Ion source (EI) temperature of $230^{\circ} \mathrm{C}$, scanning range of 28-500amu (Jiang et al., 2017; Xie et al., 2017; Lu et al., 2011; Ross et al., 2009).

\section{RESULTS AND DISCUSSION}

\section{Analysis of TGA and DTG}

In order to study the thermal decomposition of active ingredients in the bark of cornus officinalis, we conducted a TGA test on the bark. Figure 1 shows the TGA curve and DTG curve of the bark of cornus officinalis. T5 $\mathrm{wt} \%$ and $\mathrm{T} 20 \mathrm{wt} \%$ represent $5 \%$ and $20 \%$ of the weight loss, respectively (Hu and Huang, 2000; Shuyi et al., 1998; Lin, 2004). According to the graph of Fig. 1, we can obtain $\mathrm{T} 5 \mathrm{wt} \%$ and $\mathrm{T} 20 \mathrm{wt} \%$ respectively at $61^{\circ} \mathrm{C}$ and $275^{\circ} \mathrm{C}$. According to the thermogravimetric curves of the bark of cornus officinalis, the thermogravimetric analysis process is roughly divided into three stages. The first stage is $20-$ $80^{\circ} \mathrm{C}$. The main reaction at this stage is the evaporation of part of the water molecules and small molecules with lower boiling points at elevated temperatures. The quality ratio at this stage dropped from $100 \%$ to $93 \%$. The mass loss is $\mathrm{T} 7 \mathrm{wt} \%$. The DTG curve at this stage gradually increased from a lower value and reached a peak value at $48^{\circ} \mathrm{C}$. At this time, the cracking rate reached the fastest,

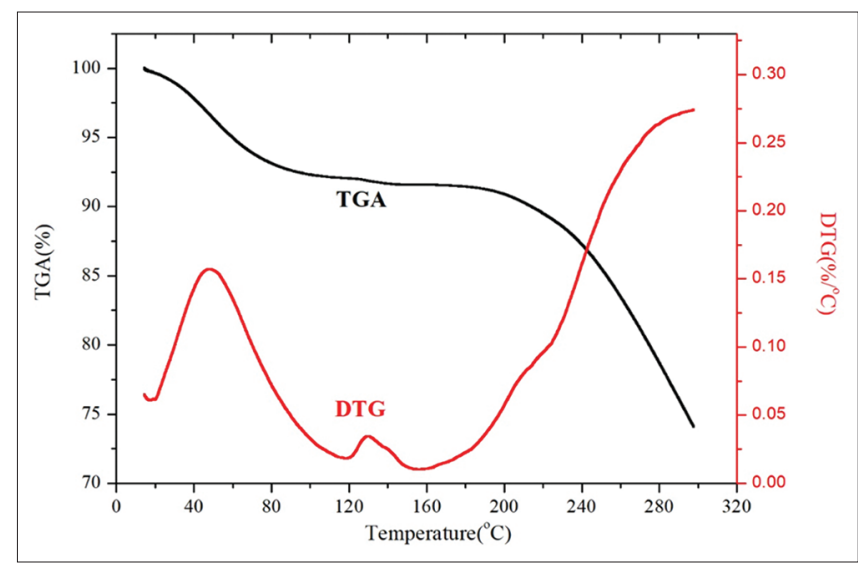

Fig 1. TGA and DTG thermal curves of $C$. officinalis bark.

and then began to gradually decline (Arsenovic et al., 2013; Chen et al., 2015; Li et al., 2013). The second phase is 80$200^{\circ} \mathrm{C}$. At this stage, a smaller amount of organic molecules begins to decompose, the mass ratio drops from $93 \%$ to $90 \%$, and the mass loss is T3wt $\%$. The DTG continued to decline at this stage, with a slight fluctuation at $120^{\circ} \mathrm{C}$, and the curve began to rebound after $160^{\circ} \mathrm{C}$. The third stage is $200-300^{\circ} \mathrm{C}$. As the temperature continues to increase, the organic components continue to undergo severe cracking and the organic components continue to dissipate. The mass ratio dropped from $90 \%$ to the final $74 \%$, and the mass loss was T16wt $\%$. At this point, the DTG curve gradually increased, indicating that the rate of cracking continued to increase during this phase. These three stages exhibited different properties and different kinetic parameters and reaction mechanisms, with a final residual mass of $74 \%$. Throughout the three phases, the weight loss in the first phase was 7\%, which was mainly due to the evaporation of some moisture molecules that remained; the quality of the second phase remained good and the weight loss was only $3 \%$; after $200^{\circ} \mathrm{C}$, the quality began to drop rapidly, and the final remaining $74 \%$. Through the TGA experimental test, the thermal decomposition of the bark of cornus officinalis below $300^{\circ} \mathrm{C}$ is described, which provides a reference for us.

The total ion chromatograms of the bark of C.officinalis samples studied via PY-GC-MS are shown in Fig 2. Highgrade resource utilization has been reported by a scholar (Wu et al., 2003; Qingzhi and Peng, 2008; Lou et al., 2018). Furthermore, the relative content of each component has been counted via area normalization. The MS data is analyzed by using the NIST standard MS map and publicly published books and papers, and then identify each component. Moreover, the analytical results of the samples are listed in Table1, respectively.

According to the results of PY-GC-MS analysis, 276 peaks were detected in Table 1, and 276 chemical constituents 
were identified. The results show that the content of more substances are as follows: Ethyne, fluoro- $(6.54 \%)$, R-(-)-Cyclohexylethylamine $(2.45 \%)$, Acetone $(2.08 \%)$, Acetic acid (4.62\%), 2-Propanone, 1-hydroxy- (1.53\%), Phenol, 2-methoxy- (1.06\%), Catechol (1.08\%), 2-Methoxy4-vinylphenol (1.67\%), Vanillin (1.03\%), trans-Isoeugenol (3.50\%), beta.-D-Glucopyranose, 1,6-anhydro- (3.03\%), 2-Propanone, 1-(4-hydroxy-3-methoxyphenyl)- (1.60\%), (E)-2,6-Dimethoxy-4-(prop-1-en-1-yl)phenol (1.46\%), Furfural (0.87\%), Creosol (0. 98\%), and n-Hexadecanoic acid $(0.99 \%)$. The main components of these detected compounds are esters, acids, phenols, anthraquinones and ketones. By analyzing the main functions and functions of different compounds, Cornus officinalis bark can be more effectively and fully utilized and exerted (Zhu et al., 2018; Xue et al., 2017; Grabowska et al., 2017; Tori et al., 2016).

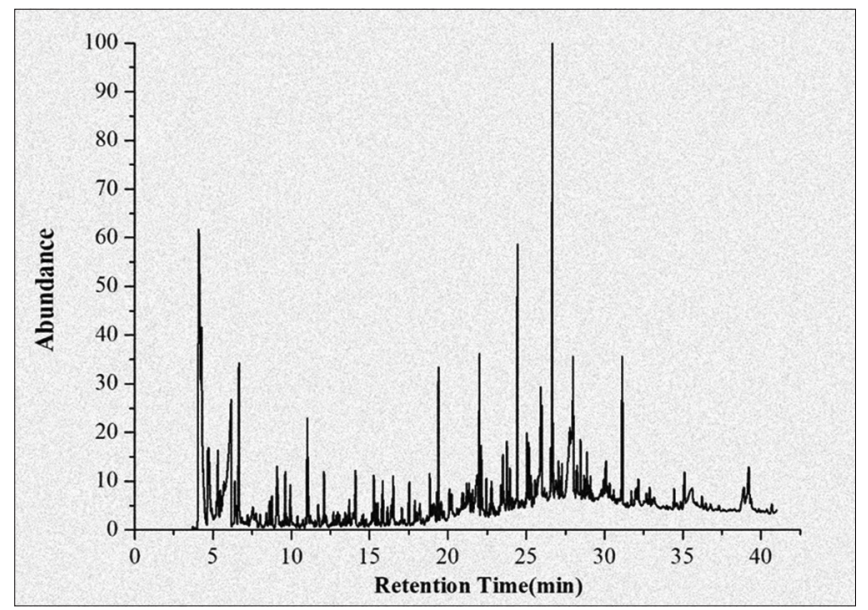

Fig 2. Total ion chromatograms of Cornus officinalis bark via PY-GC-MS.
From Table 1 can be obtained, the identified compounds can be divided into esters, alcohols, carbohydrates, tannins, iridoids, phenolics, ketones, glycosides and organic acids and so on. Among them, Furfural is a colorless, transparent, oily liquid with a special smell similar to benzaldehyde. Exposure to light and air quickly turns red-brown. Easy to evaporate with steam. Furfural is used as a raw material for organic synthesis and is widely used in the synthesis of fine chemicals such as pharmaceuticals, pesticides, veterinary drugs, dyes, spices, rubber chemicals, and preservatives. The largest area of consumption of Furfural is as a raw material for solvents and synthetic resins. Some of Furfural's derivatives have a strong bactericidal capacity and a broad spectrum of inhibition. Furfural, for example, is 5-nitrofurfural, which is condensed with semicarbazide hydrochloride to give furacilillin, a disinfectant antiseptic. At the same time, as a solvent, Furfural can selectively extract the unsaturated components from petroleum and vegetable oils. Furfural is used to extract the aromatic components in lubricating oils and diesel fuels. Improve the quality of these products (Lange et al., 2012; Palmqvist et al., 2015; Mamman et al., 2018; Eranda et al., 2011).

Another compound, phenol, is one of the main components of coal tar. Runge discovered phenol from coal tar in 1834, and Laurent produced crystalline phenol for the first time in 1841. Phenol is an important organic chemical raw material. It can be used to produce phenolic resin, bisphenol A, salicylic acid, pentachlorophenol, 2,4-D, and other chemical products and intermediates in chemical raw materials, synthetic fibers, plastics, Synthetic rubber, pharmaceuticals, pesticides, spices, dyes, coatings and oil refining industries have important applications (Vinson

Table 1: PY-GC-MS analysis of cornus officinalis bark

\begin{tabular}{lccl}
\hline No. & Retention Time $(\mathrm{min})$ & Peak area $(\%)$ & Component \\
\hline 1 & 3.70 & 0.03 & 2-Butanamine, 3,3-dimethyl- \\
2 & 3.82 & 0.00 & n-Hexylmethylamine \\
3 & 4.08 & 6.54 & Ethyne, fluoro- \\
4 & 4.23 & 0.45 & Ethyne, fluoro- \\
5 & 4.26 & 2.45 & R-(-)-Cyclohexylethylamine \\
6 & 4.36 & 0.85 & Glycidol \\
7 & 4.71 & 2.08 & Acetone \\
8 & 4.90 & 0.31 & Formic acid \\
9 & 5.00 & 0.06 & Formic acid \\
10 & 5.14 & 0.21 & 2-Propen-1-ol \\
11 & 5.23 & 0.31 & Acetaldehyde, hydroxy- \\
12 & 5.31 & 0.78 & Acetaldehyde, hydroxy- \\
13 & 5.45 & 0.53 & 2,3-Butanedione \\
14 & 5.61 & 0.40 & Pentane \\
15 & 5.70 & 0.78 & Furan, 2-methyl- \\
16 & 6.16 & 4.62 & Acetic acid \\
17 & 6.25 & 0.04 & 3-Vinyl-1-cyclobutene \\
\hline
\end{tabular}


Table 1: (Continued)

\begin{tabular}{|c|c|c|c|}
\hline No. & Retention Time (min) & Peak area (\%) & Component \\
\hline 18 & 6.31 & 0.05 & 1,3-Cyclohexadiene \\
\hline 19 & 6.38 & 0.39 & 2-Butenal, (E)- \\
\hline 20 & 6.47 & 0.25 & Methacrolein \\
\hline 21 & 6.65 & 1.53 & 2-Propanone, 1-hydroxy- \\
\hline 22 & 6.84 & 0.12 & 3-Buten-2-one, 3-methyl- \\
\hline 23 & 6.95 & 0.09 & 1-Butanol \\
\hline 24 & 7.10 & 0.11 & Acetic acid, cyclohexyl ester \\
\hline 25 & 7.21 & 0.19 & Heptane \\
\hline 26 & 7.43 & 0.19 & 1,2-Ethanediol \\
\hline 27 & 7.55 & 0.43 & Furan, 2,5-dimethyl- \\
\hline 28 & 7.75 & 0.28 & Propanoic acid \\
\hline 29 & 7.94 & 0.05 & 2-Vinylfuran \\
\hline 30 & 8.01 & 0.11 & Propanoic acid, 2-oxo-, methyl ester \\
\hline 31 & 8.08 & 0.03 & 1-Ethoxypropan-2-yl acetate \\
\hline 32 & 8.33 & 0.04 & 3-Penten-2-one, $(E)-$ \\
\hline 33 & 8.40 & 0.18 & 1H-Pyrrole, 1-methyl- \\
\hline 34 & 8.61 & 0.30 & Pyridine \\
\hline 35 & 8.75 & 0.36 & Pyrrole \\
\hline 36 & 9.08 & 0.99 & Acetic acid, methyl ester \\
\hline 37 & 9.26 & 0.10 & 2-Butenal, 2-methyl-, (E)- \\
\hline 38 & 9.43 & 0.11 & 1-Propanone, 1-(1-adamantyl)-3-dimethylamino- \\
\hline 39 & 9.62 & 0.51 & Propanoic acid, 2-oxo-, methyl ester \\
\hline 40 & 9.75 & 0.17 & 3-Octene, (Z)- \\
\hline 41 & 9.93 & 0.49 & 1,2-Propanediol, 3-(1-pyrrolidinyl)- \\
\hline 42 & 10.12 & 0.06 & 3-Pyrrolidinol \\
\hline 43 & 10.38 & 0.10 & 3-Furaldehyde \\
\hline 44 & 10.45 & 0.04 & 1H-Pyrrole, 2-ethyl- \\
\hline 45 & 10.59 & 0.02 & 3-Furanmethanol \\
\hline 46 & 10.65 & 0.03 & Pyridine, 2-methyl- \\
\hline 47 & 10.73 & 0.10 & Ethanol, 2-[(2-aminoethyl)amino]- \\
\hline 48 & 10.91 & 0.07 & Propargylamine \\
\hline 49 & 11.02 & 0.87 & Furfural \\
\hline 50 & 11.07 & 0.27 & 2-Cyclopenten-1-one \\
\hline 51 & 11.21 & 0.11 & 4-Cyclopentene-1,3-dione \\
\hline 52 & 11.29 & 0.07 & Acetamide, $\mathrm{N}$-(aminoiminomethyl)- \\
\hline 53 & 11.52 & 0.07 & 1H-Pyrrole, 3-methyl- \\
\hline 54 & 11.71 & 0.26 & 2-Furanmethanol \\
\hline 55 & 11.77 & 0.09 & 5,9-Dodecadien-2-one, 6,10-dimethyl-, (E,E))- \\
\hline 56 & 11.92 & 0.02 & Cis-bicyclo[4.2.0]octane \\
\hline 57 & 11.99 & 0.08 & Ethylbenzene \\
\hline 58 & 12.09 & 0.41 & 2-Propanone, 1-(acetyloxy)- \\
\hline 59 & 12.19 & 0.21 & 2(3H)-Furanone, 5-methyl- \\
\hline 60 & 12.46 & 0.02 & 4-Cyclopentene-1,3-dione \\
\hline 61 & 12.49 & 0.02 & Furan, 2-ethyl- \\
\hline 62 & 12.58 & 0.04 & 3-Penten-1-yne, (Z)- \\
\hline 63 & 12.69 & 0.14 & Cyclopent-4-ene-1,3-dione \\
\hline 64 & 12.86 & 0.10 & 1-Nonene \\
\hline 65 & 12.96 & 0.15 & 1,3,5,7-Cyclooctatetraene \\
\hline 66 & 13.06 & 0.14 & Pentanoic acid \\
\hline 67 & 13.14 & 0.04 & Nonane \\
\hline 68 & 13.27 & 0.05 & 2-Formylhistamine \\
\hline 69 & 13.32 & 0.07 & 1,5-Heptadiene, 3,3-dimethyl-, (E)- \\
\hline 70 & 13.45 & 0.12 & 2-Cyclopenten-1-one, 2-methyl- \\
\hline 71 & 13.57 & 0.10 & Ethanone, 1-(2-furanyl)- \\
\hline 72 & 13.70 & 0.33 & $2(5 \mathrm{H})$-Furanone \\
\hline
\end{tabular}


Table 1: (Continued)

\begin{tabular}{|c|c|c|c|}
\hline No. & Retention Time (min) & Peak area (\%) & Component \\
\hline 73 & 13.89 & 0.08 & 2-Cyclohexen-1-ol \\
\hline 74 & 13.95 & 0.11 & $\begin{array}{l}\text { 4-[2-(5-Amino-2H-1,2,3,4-tetrazol-2-yl)ethoxy]-1,2,5- } \\
\text { oxadiazol-3-amine }\end{array}$ \\
\hline 75 & 13.99 & 0.10 & 4-Pyranone, 2,3-dihydro- \\
\hline 76 & 14.10 & 0.59 & 2-Cyclopenten-1-one, 2-hydroxy- \\
\hline 77 & 14.34 & 0.02 & 2-Cyclohexen-1-one \\
\hline 78 & 14.37 & 0.04 & Pyridine, 2,4-dimethyl- \\
\hline 79 & 14.51 & 0.09 & 2(5H)-Furanone, 5-methyl- \\
\hline 80 & 14.59 & 0.10 & 2,5-Furandione, dihydro-3-methylene- \\
\hline 81 & 14.78 & 0.07 & 2-Methylbut-2-en-1-yl acetate \\
\hline 82 & 14.86 & 0.03 & 1-Methylpyrazol-3-amine \\
\hline 83 & 15.00 & 0.04 & Butanoic acid, 3-methyl-, butyl ester \\
\hline 84 & 15.09 & 0.06 & 7-Oxabicyclo[4.1.0]heptane, 1-methyl- \\
\hline 85 & 15.17 & 0.12 & 2-Butanone, 3,3-dimethyl- \\
\hline 86 & 15.27 & 0.55 & Benzaldehyde \\
\hline 87 & 15.41 & 0.19 & 2-Cyclopenten-1-one, 3-methyl- \\
\hline 88 & 15.52 & 0.16 & Pyridine, 3-ethenyl- \\
\hline 89 & 15.62 & 0.04 & 2H-Pyran-2-one \\
\hline 90 & 15.68 & 0.02 & Acrylic acid, 3-amino-3-cyano-, methyl ester \\
\hline 91 & 15.79 & 0.15 & 4-Amino-2(1H)-pyridinone \\
\hline 92 & 15.84 & 0.39 & Phenol \\
\hline 93 & 16.14 & 0.25 & 1-Decene \\
\hline 94 & 16.38 & 0.30 & 2H-Pyran-2,6(3H)-dione \\
\hline 95 & 16.50 & 0.42 & 2-Methyliminoperhydro-1,3-oxazine \\
\hline 96 & 16.56 & 0.09 & Cyclopentanecarboxylic acid, 2-ethylcyclohexyl ester \\
\hline 97 & 16.73 & 0.05 & 2-Butanone, 4-hydroxy-3-methyl- \\
\hline 98 & 16.93 & 0.04 & 1H-Pyrrole-2-carboxaldehyde \\
\hline 99 & 16.99 & 0.02 & Bicyclo[4.1.0]heptan-2-one, 6-methyl- \\
\hline 100 & 17.06 & 0.19 & 2-Cyclopenten-1-one, 2-hydroxy-3-methyl- \\
\hline 101 & 17.21 & 0.07 & 2-Butenedioic acid, 2-methyl-, (Z)- \\
\hline 102 & 17.36 & 0.01 & Benzene, 1-ethyl-4-methyl- \\
\hline 103 & 17.43 & 0.02 & Benzene, 2-propenyl- \\
\hline 104 & 17.53 & 0.45 & 2-Cyclopenten-1-one, 2-hydroxy-3-methyl- \\
\hline 105 & 17.65 & 0.02 & Benzyl alcohol \\
\hline 106 & 17.72 & 0.02 & Ethanone, 1-(2-methyl-1-cyclopenten-1-yl)- \\
\hline 107 & 17.89 & 0.23 & Hydantoin, 5,5-dimethyl-2-thio-, \\
\hline 108 & 18.01 & 0.19 & 4-Methyl-5H-furan-2-one \\
\hline 109 & 18.21 & 0.40 & 1,3-Dioxol-2-one,4,5-dimethyl- \\
\hline 110 & 18.42 & 0.06 & 2-Cyclopenten-1-one, 2-hydroxy-3,4-dimethyl- \\
\hline 111 & 18.55 & 0.02 & endo-Borneol \\
\hline 112 & 18.60 & 0.03 & Ethanone, 1-(1H-pyrrol-2-yl)- \\
\hline 113 & 18.68 & 0.10 & 1-Nonene \\
\hline 114 & 18.77 & 0.06 & Benzaldehyde, 2-methyl- \\
\hline 115 & 18.84 & 0.49 & p-Cresol \\
\hline 116 & 18.99 & 0.20 & 2-Pentyne \\
\hline 117 & 19.12 & 0.27 & Heptanoic acid \\
\hline 118 & 19.16 & 0.08 & Heptanoic acid \\
\hline 119 & 19.29 & 0.20 & 2,5-Piperazinedione, 3-methyl-6-(1-methylethyl)- \\
\hline 120 & 19.39 & 1.06 & Phenol, 2-methoxy- \\
\hline 121 & 19.52 & 0.14 & Pyridine, 5-ethyl-2-methyl- \\
\hline 122 & 19.57 & 0.25 & $\begin{array}{l}\text { Carbamic acid, (2-hydroxy-1-methylethyl)-, } \\
\text { 1,1-dimethylethyl ester, (s)- }\end{array}$ \\
\hline 123 & 19.74 & 0.14 & 2-Furanmethanol \\
\hline 124 & 19.98 & 0.06 & Benzofuran, 2-methyl- \\
\hline 125 & 20.10 & 0.39 & Maltol \\
\hline
\end{tabular}


Table 1: (Continued)

\begin{tabular}{|c|c|c|c|}
\hline No. & Retention Time (min) & Peak area $(\%)$ & Component \\
\hline 126 & 20.19 & 0.12 & 2(1H)-Pyridinone \\
\hline 127 & 20.24 & 0.33 & 2-Cyclopenten-1-one, 3-ethyl-2-hydroxy- \\
\hline 128 & 20.34 & 0.06 & trans-Sinapyl alcohol \\
\hline 129 & 20.39 & 0.10 & 2,5-Piperazinedione, 3-methyl- \\
\hline 130 & 20.58 & 0.28 & trans-Sinapyl alcohol \\
\hline 131 & 20.72 & 0.16 & Benzyl nitrile \\
\hline 132 & 20.79 & 0.15 & trans-Sinapyl alcohol \\
\hline 133 & 20.86 & 0.11 & Phenol, 2,3-dimethyl- \\
\hline 134 & 20.92 & 0.29 & 4H-Pyran-4-one, 2,3-dihydro-3,5-dihydroxy-6-methyl- \\
\hline 135 & 21.00 & 0.24 & 2H-Pyran-2-one, tetrahydro- \\
\hline 136 & 21.06 & 0.16 & 2a,4a,6a,6b-Tetrahydrocyclopenta[cd]pentalene \\
\hline 137 & 21.11 & 0.10 & trans-Sinapyl alcohol \\
\hline 138 & 21.20 & 0.48 & 1,3-Cyclopentanedione, 4-hydroxy-2-methyl- \\
\hline 139 & 21.29 & 0.21 & Phenol, 3-ethyl- \\
\hline 140 & 21.35 & 0.35 & Dehydromevalonic lactone \\
\hline 141 & 21.41 & 0.25 & 2-Cyclohexen-1-one, 4,4-dimethyl- \\
\hline 142 & 21.58 & 0.43 & Octanoic acid \\
\hline 143 & 21.64 & 0.10 & E-7-Tetradecenol \\
\hline 144 & 21.82 & 0.74 & 1,4,2,5 Cyclohexanetetrol \\
\hline 145 & 21.88 & 0.41 & 2-Vinyl-9-[.beta.-d-ribofuranosyl]hypoxanthine \\
\hline 146 & 21.91 & 0.30 & Butanal, 3-hydroxy- \\
\hline 147 & 22.01 & 0.98 & Creosol \\
\hline 148 & 22.12 & 1.08 & Catechol \\
\hline 149 & 22.27 & 0.08 & Ethanethioic acid, S-phenyl ester \\
\hline 150 & 22.31 & 0.08 & 6-Hydroxymethyl-5-methyl-bicyclo[3.1.0]hexan-2-one \\
\hline 151 & 22.46 & 0.35 & Benzofuran, 2,3-dihydro- \\
\hline 152 & 22.53 & 0.09 & Pyridine, 2,3-dimethyl- \\
\hline 153 & 22.67 & 0.21 & Z-8-Methyl-9-tetradecenoic acid \\
\hline 154 & 22.78 & 0.62 & 5-Hydroxymethylfurfural \\
\hline 155 & 22.89 & 0.06 & Methanol, (4-carboxymethoxy)benzoyl- \\
\hline 156 & 22.95 & 0.15 & 2-Coumaranone \\
\hline 157 & 23.04 & 0.09 & 4-Pentadecyne, 15-chloro- \\
\hline 158 & 23.11 & 0.08 & 3-Buten-2-ol, 4-(2,6,6-trimethyl-2-cyclohexen-1-yl)-, (3E)- \\
\hline 159 & 23.16 & 0.09 & 2-Cyclohexen-1-one, 4-(1-methylethyl)- \\
\hline 160 & 23.28 & 0.14 & Estra-1,3,5(10)-trien-17.beta.-ol \\
\hline 161 & 23.39 & 0.44 & 1,2-Benzenediol, 3-methyl- \\
\hline 162 & 23.51 & 0.51 & 1,2-Benzenediol, 3-methoxy- \\
\hline 163 & 23.56 & 0.43 & 2,2-Dimethyl-3-vinyl-bicyclo[2.2.1]heptane \\
\hline 164 & 23.71 & 0.07 & Z-10-Pentadecen-1-ol \\
\hline 165 & 23.78 & 0.56 & Phenol, 4-ethyl-2-methoxy- \\
\hline 166 & 23.85 & 0.20 & 1-Tridecene \\
\hline 167 & 23.97 & 0.79 & 1,2-Benzenediol, 4-methyl- \\
\hline 168 & 24.12 & 0.15 & Indole \\
\hline 169 & 24.17 & 0.16 & 4,6-Dioxadodecane \\
\hline 170 & 24.24 & 0.09 & Kessane \\
\hline 171 & 24.29 & 0.19 & Oxaceprol \\
\hline 172 & 24.35 & 0.22 & $\begin{array}{l}\text { 1,3-Dimethyl-4-(tetramethyl-1,3,2-dioxaborolan-2-yl) } \\
\text { pyrazole }\end{array}$ \\
\hline 173 & 24.45 & 1.67 & 2-Methoxy-4-vinylphenol \\
\hline 174 & 24.57 & 0.31 & $\begin{array}{l}\text { 2,3-Dioxabicyclo[2.2.2]oct-7-en-5-one, 1-(3-oxo-1-butenyl)- } \\
\text { 6,6,7-trimethyl }\end{array}$ \\
\hline 175 & 24.71 & 0.20 & Acetamide, N-(4-fluorophenyl)-2-methoxy- \\
\hline 176 & 24.74 & 0.30 & 2(equat)-Methyl-trans-decahydroquinol-4-one \\
\hline 177 & 24.88 & 0.16 & endo-1,5,6,7-Tetramethylbicyclo[3.2.0]hept-6-en-3-ol \\
\hline 178 & 24.97 & 0.27 & Bicyclo[2.2.1]heptane-1-carbonyl chloride, 2-exo-chloro- \\
\hline 179 & 25.05 & 0.73 & Phenol, 2,6-dimethoxy- \\
\hline
\end{tabular}


Table 1: (Continued)

\begin{tabular}{|c|c|c|c|}
\hline No. & Retention Time (min) & Peak area $(\%)$ & Component \\
\hline 180 & 25.17 & 0.60 & Eugenol \\
\hline 181 & 25.23 & 0.14 & cis-11-Hexadecenal \\
\hline 182 & 25.27 & 0.20 & Phenol, 3,4-dimethoxy- \\
\hline 183 & 25.33 & 0.34 & Phenol, 2-methoxy-4-propyl- \\
\hline 184 & 25.43 & 0.28 & 1,2-15,16-Diepoxyhexadecane \\
\hline 185 & 25.57 & 0.58 & 1,3-Benzenediol, 4-ethyl- \\
\hline 186 & 25.66 & 0.27 & Tetradecane \\
\hline 187 & 25.74 & 0.36 & Indole, 3-methyl- \\
\hline 188 & 25.78 & 0.29 & 1,2,3-Benzenetriol \\
\hline 189 & 25.82 & 0.14 & 1,2,3-Benzenetriol \\
\hline 190 & 25.86 & 0.40 & 1,2,3-Benzenetriol \\
\hline 191 & 25.93 & 1.03 & Vanillin \\
\hline 192 & 25.99 & 0.71 & Phenol, 2-methoxy-4-(1-propenyl)- \\
\hline 193 & 26.10 & 0.18 & $1,2,4$-Benzenetriol \\
\hline 194 & 26.16 & 0.39 & $\begin{array}{l}\text { 2,3-Dioxabicyclo[2.2.2]oct-7-en-5-one, 1-(3-oxo-1-butenyl)- } \\
\text { 6,6,7-trimethyl }\end{array}$ \\
\hline 195 & 26.30 & 0.47 & (2,4-Dioxo-1,3-dihydropyrimidin-5-yl)acetic acid \\
\hline 196 & 26.45 & 0.14 & $\begin{array}{l}\text { 2,2,2-Trifluoro-N-[4-(2,2,2-trifluoro-acetylamino)-butyl]- } \\
\text { acetamide }\end{array}$ \\
\hline 197 & 26.48 & 0.13 & 3,4-Dimethyl-o-phenylenediamine \\
\hline 198 & 26.57 & 0.63 & 3,5-Dimethoxy-4-hydroxytoluene \\
\hline 199 & 26.67 & 3.50 & trans-Isoeugenol \\
\hline 200 & 26.73 & 0.35 & E-9-Tetradecenoic acid \\
\hline 201 & 26.83 & 0.36 & 4-Methoxycinnamaldehyde \\
\hline 202 & 26.88 & 0.13 & 2-Cyano-2-[2-cyclopropyldiazen-1-yl]ethanethioamide \\
\hline 203 & 26.92 & 0.31 & 1-Pentadecene \\
\hline 204 & 26.98 & 0.33 & Ethyl 9-heptadecenoate \\
\hline 205 & 27.07 & 0.50 & 1-Pentadecene \\
\hline 206 & 27.18 & 0.33 & Pentadecane \\
\hline 207 & 27.29 & 0.68 & Ethanone, 1-(3-hydroxy-4-methoxyphenyl)- \\
\hline 208 & 27.80 & 3.03 & Beta.-D-Glucopyranose, 1,6-anhydro- \\
\hline 209 & 27.86 & 0.65 & Beta.-D-Glucopyranose, 1,6-anhydro- \\
\hline 210 & 27.93 & 0.75 & D-Allose \\
\hline 211 & 27.99 & 1.60 & 2-Propanone, 1-(4-hydroxy-3-methoxyphenyl)- \\
\hline 212 & 28.10 & 0.31 & Oxacyclotetradecane-2,11-dione, 13-methyl- \\
\hline 213 & 28.20 & 0.41 & Dodecanoic acid \\
\hline 214 & 28.27 & 0.67 & Nonanoic acid, 1-methylethyl ester \\
\hline 215 & 28.48 & 0.73 & 2,3,5,6-Tetrafluoroanisole \\
\hline 216 & 28.58 & 0.16 & 2-Dodecen-1-yl(-)succinic anhydride \\
\hline 217 & 28.64 & 0.23 & Bicyclo(3.3.1)nonane-2,6-dione \\
\hline 218 & 28.70 & 0.48 & Cyclotetradecane \\
\hline 219 & 28.81 & 0.23 & Nonadecane, 9-methyl- \\
\hline 220 & 28.88 & 0.54 & .alpha.-Amino-3'-hydroxy-4'-methoxyacetophenone \\
\hline 221 & 28.94 & 0.53 & Butyrovanillone \\
\hline 222 & 29.12 & 0.55 & Phenol, 2,6-dimethoxy-4-(2-propenyl)- \\
\hline 223 & 29.24 & 0.25 & 4-Propyl-1,1'-diphenyl \\
\hline 224 & 29.41 & 0.32 & Estra-1,3,5(10)-trien-17.beta.-ol \\
\hline 225 & 29.47 & 0.17 & n-PROPYL DECYL ETHER \\
\hline 226 & 29.54 & 0.26 & Oxacyclotetradecan-2-one \\
\hline 227 & 29.66 & 0.26 & 2,2-Dimethyl-propyl S-benzene-thiosulfinate \\
\hline 228 & 29.74 & 0.28 & 9-Hexadecenoic acid \\
\hline 229 & 29.81 & 0.18 & $\begin{array}{l}\text { Imidazo[4,5-e]-1,4-diazepin-8-one, 1,4,5,6,7,8-hexahydro- } \\
\text { 1,4-dimethyl- }\end{array}$ \\
\hline 230 & 29.82 & 0.21 & Palmitoleic acid \\
\hline 231 & 29.96 & 0.57 & Tridecanoic acid \\
\hline 232 & 30.11 & 0.94 & Benzenepropanol, 4-hydroxy-3-methoxy- \\
\hline
\end{tabular}


Table 1: (Continued)

\begin{tabular}{|c|c|c|c|}
\hline No. & Retention Time (min) & Peak area (\%) & Component \\
\hline 233 & 30.22 & 0.36 & Z,E-2,13-Octadecadien-1-ol \\
\hline 234 & 30.33 & 0.56 & Benzaldehyde, 4-hydroxy-3,5-dimethoxy- \\
\hline 235 & 30.46 & 0.28 & Cyclopentadecanone, 2-hydroxy- \\
\hline 236 & 30.60 & 0.47 & 1-Heptadecene \\
\hline 237 & 30.74 & 0.25 & 9-Hexadecenoic acid \\
\hline 238 & 30.85 & 0.30 & 5-Amino-2-thiocyanoacetophenone \\
\hline 239 & 30.93 & 0.09 & $\begin{array}{l}\text { 2H-1-Benzopyran-3-ol, 2-(3,4-dimethoxyphenyl)-3,4- } \\
\text { dihydro-5,7-dimethoxy-, (2R-cis)- }\end{array}$ \\
\hline 240 & 31.01 & 0.22 & $\begin{array}{l}\text { 3,9-Epoxytricyclo[4.2.1.1(2,5)]dec-7-en-10-ol, } \\
\text { 9,10-dimethyl- }\end{array}$ \\
\hline 241 & 31.14 & 1.46 & (E)-2,6-Dimethoxy-4-(prop-1-en-1-yl)phenol \\
\hline 242 & 31.46 & 0.19 & Cyanoacetic acid, dodecyl ester \\
\hline 243 & 31.65 & 0.15 & $\begin{array}{l}6 \mathrm{H}-1,2,5-O x a d i a z o l o[3,4-E] \text { indole-6,8a-diol, 4,5,5a,7,8,8a- } \\
\text { hexahydro-, 3-oxide }\end{array}$ \\
\hline 244 & 31.72 & 0.29 & I-Norleucine, N-isobutoxycarbonyl-, heptadecyl ester \\
\hline 245 & 31.81 & 0.11 & $\begin{array}{l}\text { 8',10'-Dioxaspiro[cyclopropane-1,5'-tricyclo[5.2.1.0 } 0\{2,4\}] \\
\text { decane]-6'-one }\end{array}$ \\
\hline 246 & 31.99 & 0.53 & E-9-Tetradecenoic acid \\
\hline 247 & 32.16 & 0.58 & Tetradecanoic acid \\
\hline 248 & 32.26 & 0.23 & $\mathrm{~N}$-[p-Nitrobenzoyl]anthranilic acid \\
\hline 249 & 32.52 & 0.27 & 1,5-Dodecadiene \\
\hline 250 & 32.68 & 0.48 & cis-1-Chloro-9-octadecene \\
\hline 251 & 32.89 & 0.43 & 2-Propanone, 1,1-diphenyl- \\
\hline 252 & 33.02 & 0.18 & 1-Nonadecene \\
\hline 253 & 33.19 & 0.42 & 13-Octadecenal, (Z)- \\
\hline 254 & 33.73 & 0.07 & 1-Octadecene \\
\hline 255 & 33.97 & 0.14 & Methyl 13-methyl-icosanoate \\
\hline 256 & 34.17 & 0.11 & 9-Ethoxy-10-oxatricyclo[7.2.1.0(1,6)]dodecan-11-one \\
\hline 257 & 34.45 & 0.32 & Ethanone, 1-(4-hydroxy-3,5-dimethoxyphenyl)- \\
\hline 258 & 34.63 & 0.28 & 13-Octadecenal, (Z)- \\
\hline 259 & 34.88 & 0.20 & Cyclopentadecanone, 2-hydroxy- \\
\hline 260 & 35.12 & 0.62 & Pentadecanoic acid \\
\hline 261 & 35.25 & 0.13 & Octadecanoic acid \\
\hline 262 & 35.51 & 0.74 & Octadecanoic acid \\
\hline 263 & 35.59 & 0.51 & Octadecanoic acid \\
\hline 264 & 35.92 & 0.15 & 18-Nonadecenoic acid \\
\hline 265 & 36.24 & 0.23 & 1-Octadecene \\
\hline 266 & 36.47 & 0.15 & Tricosane \\
\hline 267 & 36.78 & 0.17 & 1,1'-Biphenyl, 4-methyl- \\
\hline 268 & 37.31 & 0.09 & 7-Hexadecenoic acid, methyl ester, (Z)- \\
\hline 269 & 38.91 & 0.68 & Hexadecenoic acid, Z-11- \\
\hline 270 & 39.05 & 0.21 & Oxacycloheptadecan-2-one \\
\hline 271 & 39.23 & 0.99 & n-Hexadecanoic acid \\
\hline 272 & 39.35 & 0.23 & 5-Undecene \\
\hline 273 & 39.85 & 0.06 & 9-Octadecenoic acid, $(\mathrm{E})$ - \\
\hline 274 & 40.37 & 0.05 & 2-Dodecen-1-yl(-)succinic anhydride \\
\hline 275 & 40.39 & 0.03 & Cyclopentadecanone, 2-hydroxy- \\
\hline 276 & 40.69 & 0.12 & 1-Docosene \\
\hline
\end{tabular}

et al., 2001; Bystrom et al., 2010). Phenol can denature proteins, so it has a bactericidal effect and shows strong antibacterial activity against many microorganisms, certain fungi, and viruses. Dilute solution phenol can be used as a disinfectant antiseptic and was used in surgery (Ogunrinola et al., 1996; Wang, 2007). According to statistics, in the $21^{\text {st }}$ century, the demand for phenol in major regions such as the United States, Western Europe, and Japan maintained an average annual growth rate of $4.2 \%$.

Maltol, is a white to slightly yellow needle crystal or crystalline powder, with a special aroma of coke cream, and a dilute solution with a strawberry flavor. It is mainly used in the food industry to formulate strawberry, coffee, 
malt, nuts, herbs and various fruit flavors. Maltol is also an excellent material for the smooth coating of photographic film to prevent spots and streaks. The skin care cosmetics formulated therewith have the effect of inhibiting melanin growth and whitening the skin (Thompson et al., 2006; Ferreira et al., 2003; Ni et al., 2005; Yasumoto et al., 2004; Singh et al., 2007; Zhang et al., 2000; Liu, 2017; Kmita et al., 2018; Hoque et al., 2018; Khan et al., 2018; Cui et al., 2018).

\section{CONCLUSION}

From the above studies, it can be seen that the TGA-DTG analysis showed that the weight loss process of cornus officinalis bark was divided into three stages. The first stage is $20-80^{\circ} \mathrm{C}$. The reason for the decline in this part of the curve is that the water molecules contained in the leaves and small molecules with relatively low boiling points evaporate as the temperature rises; the second stage is $80-200^{\circ} \mathrm{C}$, during which some organic molecules begin to decompose, and the mass ratio is from $93 \%$ decline to $90 \%$; final stage at $200-300^{\circ} \mathrm{C}$. At this stage, as the temperature continues to rise, the organic components of the component undergo severe cracking decomposition and combustion of other components, the mass ratio drops from $90 \%$ to the final $74 \%$, and the mass loss is $\mathrm{T} 16 \mathrm{wt} \%$.

In the PY-GC-MS test, 276 peaks were detected in the bark of cornus officinalis and 276 chemical components were identified. The identified compounds can be classified into esters, acids, phenols, tannins, iridoids, soaps, ketones, and glycosides. Among them, Furfural is not only an important organic material in medicine and industry, but also can be used as a solvent to selectively extract unsaturated components from petroleum and vegetable oils. As an important chemical material, phenol can denature protein to make it have a certain bactericidal effect. Maltol is widely used in a variety of fruit flavors in the food industry due to its unique creamy flavor characteristics. At the same time, it also has a good effect of inhibiting melanin growth and whitening the skin.

Through the research and analysis of the volatiles of cornus officinalis bark, we can clearly understand the organic substances it contains, provide reference for the development of medical or industrial food, and provide fuller and more extensive value for cornus officinalis bark, and provides better help.

\section{ACKNOWLEDGMENTS}

The Hunan Science Fund for Distinguished Young Scholars (16JJ1028), The authors acknowledge financial support by the new century excellent talent support program (NCET-12-0725), the Major scientific and technological achievements transformation projects of strategic emerging industries in Hunan Province (2016GK4045), Innovation and venture capital investment project in Hunan(2017GK5027), and the Academician reserve personnel training plan of lift engineering technical personnel of Hunan Science and Technology Association (2017TJ-Y10). Meanwhile, the authors are so thankful to Emate(www.emate.ac.cn) for language service.

\section{REFERENCES}

Arsenović, M., L. Pezo and S. Stanković. 2013. Sensitivity analysis of mathematical models for final product properties: Link to DTG curve. Ceram. Int. 39(6): 6277-6285.

Byström, A., R. Claesson and G. Sundqvist. 2010. The antibacterial effect of camphorated paramonochlorophenol, camphorated phenol and calcium hydroxide in the treatment of infected root canals. Endod. Dent. Traumatol. 1(5): 170-175.

Cao, Y. and X. Lei. 2013. The influence of total saponin of Cornus officinalis sieb.et zucc. on proliferation and apoptosis of K562 cells. J. Xiangnan Univ. 15(3): 18-21.

Chen, C. D., B. Jin and R. F. Peng. 2015. Study on the synthesis and thermal stability of the poly (glycidyl azide)-b-poly(vinyl acetate azide). J. Saf. Environ. 15(02): 138-143.

Chen, Y., Y. Wu and X. Gan. 2016. Iridoid glycoside from Cornus officinalis, ameliorated diabetes mellitus-induced testicular damage in male rats: Involvement of suppression of the AGEs/ RAGE/p38 MAPK signaling pathway. J. Ethnopharmacol. 194: 850-860.

Cui, L., N. He, X. Zhang, S. Li, Y. Zhang and W. Kang. 2018. Dynamic change of secondary metabolites and spectrum-effect relationship of Malus halliana koehne flowers during blooming. Open Chem. 16(1): 362-370.

El Toum, G. A. 2018. Productivity of pure stands and intercropped Forage sorghum and hyacinth bean. Malays. J. Sustain. Agric. 2(1): 5-6.

Eranda, N., R. Yuriy and M. Manuel. 2011. "One-pot" Synthesis of 5-(Hydroxymethyl)furfural from carbohydrates using tin-beta zeolite. ACS Catal. 1: 408-410.

Ferreira, V., I. Jarauta and R. López. 2003. Quantitative determination of sotolon, maltol and free furaneol in wine by solid-phase extraction and gas chromatography-ion-trap mass spectrometry. J. Chromatogr. A. 1010(1): 95-103.

Gornicka, B. and L. Gorecki. 2010. TGA/DTG/DSC investigation of thermal ageing effects on polyamide-imide enamel. J. Therm. Anal. Calorim. 101(2): 647-650.

Grabowska, B., K. Kaczmarska and A. Bobrowski. 2017. TGDTG-DSC, FTIR, DRIFT and Py-GC-MS studies of thermal decomposition for poly (sodium acrylate)/dextrin (PAANa/D) new binder BioCo3. J. Casting Mater. Eng. 1(1): 27.

Han, Z., J. Zhang and Y. Zhao. 2005. Comparing the effect of seven extraction methods of the total saponins from Cornus officinalis sieb. et Zucc. J. Zhengzhou Univ. 40(3): 466-468.

Hasan, M. M. 2018. Bioaugmentation approach in rhizospheric microbiome research: A lesson from arsenic remediation. Malays. J. Halal Res. 1(1): 15-16.

He, K., S. Song and Z. Zou. 2016. The hypoglycemic and synergistic effect of loganin, morroniside, and ursolic acid isolated from the fruits of Cornus officinalis. Phytother. Res. 30(2): 283-291. 
Hong, W. U., H. Liang and Y. H. Liu. 2003. Extraction and determination of total saponins from Cornus officinalis Sieb. et Zucc. J. Fourth Mil. Med. Univ. 24(5): 430-432.

Hoque, M. A., F. M. Hassan, A. M. Jauhar, G. Jiang, M. Pritzker, J. Choi, S. Knights, S. Ye and Z. Chen. 2018. Web-Like 3D architecture of pt nanowires and sulfur-doped carbon nanotube with superior electrocatalytic performance. ACS Sustain. Chem. Eng. 6(1): 93-98.

Howlader, M. H. K., M. N. Islam, S. Biswas, M. E. Uddin, A. Shila, M. Z. Haque and N. Mahmud. 2018. Salt tolerance of chili genotypes during germination and seedling growth. Malays. J. Halal Res. 1(2): 1-7.

Hsu, J. H., Y. C. Wu and I. M. Liu. 2006. Release of acetylcholine to raise insulin secretion in wistar rats by oleanolic acid, one of the active principles contained in Cornus officinalis. Neurosci. lett. 404(1): 112-116.

Hu, S. Y. and B. Z. Huang. 2000. Analysis of DTA/TG curve of phenolic resin dipped wood powder. Chem. Ind. For. Prod. 20(1): 47-51.

Huang, J., Y. Zhang and L. Dong. 2017. Ethnopharmacology, phytochemistry, and pharmacology of Cornus officinalis, Sieb. et Zucc. J. Ethnopharmacol. 213: 280-301.

Jang, S. E., J. J. Jeong and S. R. Hyam. 2014. Ursolic acid isolated from the seed of Cornus officinalis ameliorates colitis in mice by inhibiting the binding of lipopolysaccharide to toll-like receptor 4 on macrophages. J. Agric. Food Chem. 62(40): 9711-21.

Jiang, S. C., S. B. Ge and M. Z. Wang. 2017. Molecules and functions of rosewood: Dalbergia Stevenson. Arabian J. Chem. 11: 782-792.

Khan, F., M. I. Khan, S. Khan, M. A. U. Zaman and H. Rasheed. 2018. Evaluation of agronomic traits for yield and yield components in wheat genotypes with respect to planting dates. Malays. J. Sustain. Agric. 2(1): 7-11.

Khan, M. M. H. 2018. Occurrence, distribution, host preference and damage severity of red pumpkin beetle-a review. Malays. J. Halal Res. 1(1): 3-9.

Khan, S. A., S. M. Imam, A. Ahmad, S. H. Basha and A. Husain. 2018. Synthesis, molecular docking with cox 1 and li enzyme, admet screening and in vivo anti-inflammatory activity of oxadiazole, thiadiazole and triazole analogs of felbinac. J. Saudi Chem. Soc. 22(4): 469-484.

Kmita, A., C. Fischer, K. Hodor, M. Holtzer and A. Roczniak. 2018. Thermal decomposition of foundry resins: A determination of organic products by thermogravimetry-gas chromatographymass spectrometry (Tg-Gc-Ms). Arabian J. Chem. 11(3): 380-387.

Kök, M. V., M. A. Varfolomeev and D. K. Nurgaliev. 2016. Thermal characterization of crude oils in the presence of limestone matrix by TGA-DTG-FTIR. J. Pet. Sci. Eng. 99: 1.

Lange, J. P., H. E. Van D and J. Van Buijtenen. 2012. Furfural--a promising platform for lignocellulosic biofuels. Chemsuschem. 5(1): 150-166.

Li, Y. L., L. P. Mao and C. F. Xu. 2013. A preliminary study of the carbon-based solid acid preparation process. Adv. Mater. Res. 734-737: 2236-9.

Lin, Q. 2004. Analysis of DTA/TG curve of wood with phosphoric acid and zinc chloride. Sci. Silvae Sinicae. 40(1): 142-147.

Liu, Z. 2017. China's strategy for the development of renewable energies. Energy Sources B Econ. Plan. Policy. 12(11): 971-975.

Liu, Z. 2017. Teaching reform of business statistics in college and university. Eurasia J. Math. Sci. Technol. Educ. 13(10): 6901-6907.

Lou, J., J. Chen and C. Ni. 2018. Molecules and functions of rosewood: Pterocarpus cambodianus. Arabian J. Chem. 11: 763-770.

Lu, Q., X. C. Yang and C. Q. Dong. 2011. Influence of pyrolysis temperature and time on the cellulose fast pyrolysis products:
Analytical Py-GC/MS study. J. Analyt. Appl. Pyrolysis. 92(2): 430-438.

Ma, W., K. J. Wang and C. S. Cheng. 2014. Bioactive compounds from Cornus officinalis, fruits and their effects on diabetic nephropathy. J. Ethnopharmacol. 153(3): 840-845.

Mamman, A. S., J. M. Lee and Y. C. Kim. 2010. Furfural: Hemicellulose/xylose-derived biochemical. Biofuel. Bioprod. Biorefin. 2(5): 438-454.

Miyazawa, M. and H. Kameoka. 2014. Volatile flavor components of CORNI FRUCTUS (Cornus officinalis Sieb. et Zucc.). J. Agric. Chem. Soc. Japan. 53(12): 3337-3340.

Miyazawa, M. and H. Kameoka. 2014. Volatile flavor components of CORNI FRUCTUS (Cornus officinalis Sieb. et Zucc.). J. Agric. Chem. Soc. Japan. 53(12): 3337-3340.

Ni, Y. N., G. W. Zhang and S. Kokot. 2005. Simultaneous spectrophotometric determination of maltol, ethyl maltol, vanillin and ethyl vanillin in foods by multivariate calibration and artificial neural networks. Food Chem. 89(3): 465-473.

Ogunrinola, O. A., I. J. Jeon and D. Y. C. Fung. 1996. Effects of mode of sterilization on the recovery of phenolic antioxidants in laboratory media assessed by nonderivatizing gas chromatography-mass spectrometry. J. Food Prot. 59(12): 1322-1326.

Palmqvist, E., J. S. Almeida and B. Hahn-Hägerdal. 2015. Influence of furfural on anaerobic glycolytic kinetics of Saccharomyces cerevisiae in batch culture. Biotechnol. Bioeng. 62(4): 447-454.

Palmqvist, E., H. Grage and N. Q. Meinander. 2015. Main and interaction effects of acetic acid, furfural, and p-hydroxybenzoic acid on growth and ethanol productivity of yeasts. Biotechnol. Bioeng. 63(1): 46-55.

Qian, D. S., Y. F. Zhu and Q. Zhu. 2001. Effect of alcohol extract of Cornus officinalis Sieb. et Zucc on GLUT4 expression in skeletal muscle in Type 2 (non-insulin-dependent) diabetic mellitus rats. China J. Chin. Mater. Med. 26(12): 859-862.

Qingzhi, M. A. and W. Peng. 2008. Py-GC/MS Study on High-Grade Resource Recovery of Phyllostachys heterocycla. Conference on Regional Economy and Sustainable Development.

Ren, Y. S., X. U. Feng-Qing and M. A. Feng-Ai. 2016. Study on the chemical constituents from the fruit of Cornus officinalis. Guangzhou Chem. Ind. 44(8): 76-77.

Ross, A. B., K. Anastasakis and M. Kubacki. 2009. Investigation of the pyrolysis behaviour of brown algae before and after pretreatment using PY-GC/MS and TGA. J. Anal. Appl. Pyrolysis. 85(1): 3-10.

Shuyi, A. H., Z. Zhang and B. L. Huang. 1998. Analysis of DTA/TG curve of wood flame retardant of inorganic boride series. J. Fujian Coll. For. 18: 163-166.

Singh, G., S. Maurya and M. P. Delampasona. 2007. A comparison of chemical, antioxidant and antimicrobial studies of cinnamon leaf and bark volatile oils, oleoresins and their constituents. Food Chem. Toxicol. 45(9): 1650-1661.

Telang, N. T., G. Li and D.W. Sepkovic. 2012. Anti-proliferative effects of Chinese herb Cornus officinalis in a cell culture model for estrogen receptor-positive clinical breast cancer. Mol. Med. Rep. 5(1): 22.

Telang, N. T., H. B. Nair and G. Wong. 2016. Growth inhibitory effects of Cornus officinalis on a model for triple negative breast cancer. Cancer Res. 76: P3-09-04-P3-09-04.

Thompson, K. H., C. A. Barta and C. Orvig. 2006. Metal complexes of maltol and close analogues in medicinal inorganic chemistry. Chem. Soc. Rev. 35(6): 545-556.

Torri, C., D. L. Barreiro and R. Conti. 2016. Fast procedure for the analysis of hydrothermal liquefaction biocrude with stepwise Py-GC-MS and data interpretation assisted by means of 
non-negative matrix factorization. Energy Fuels. 30(2): 11351144.

Trivedi, M. K., K. K. Sethi and P. Panda. 2017. Sodyum selenat'ın XRD, PSD, DSC, TGA/DTG, UV-VIS ve FT-IR yöntemleri ile fizikokimyasal, termal ve spektroskopik karakterizasyonu. Marmara Pharm. J. 21(2): 311-311.

Vinson, J. A., Y. Hao and X. H. Su. 1998. Phenol antioxidant quantity and quality in foods: Vegetables. J. Agric. Food Chem. 46(9): 3630-3634.

Vinson, J. A., X. Su and L. Zubik. 2001. Phenol antioxidant quantity and quality in foods: Fruits. J Agric Food Chem. 49(11): 5315-21.

Wang, H. L. 2007. Clinical evaluation on effects of cidex, formocresol and camphor phenol for root canal sterilization. China Pract. Med. 2(15): 13-15.

Wang, R., S. M. Wang and S. W. Liang. 2007. Separation and determination of oleanolic acid and ursolic acid from Cornus officinalis by capillary electrophoresis. J. Chin. Med. Mater. 30(8): 946.

Wu, Y. Q., W. X. Peng and X. G. Li. 2009. Py-GC/MS Analysis on Biomedical Components of Phyllostachys pubescens.
International Conference on Bioinformatics and Biomedical Engineering, p. 1-3.

Xie, Y., S. Ge and S. Jiang. 2017. Study on biomolecules in extractives of Camellia oleifera, fruit shell by GC-MS. Saudi J. Biol. Sci. 25(2):234-236.

Xue, J., J. Zhuo and M. Liu. 2017. Synergetic effect of co-pyrolysis of cellulose and PP over an all-silica mesoporous catalyst MCM-41 using TG-FTIR and Py-GC-MS. Energy Fuels. 31: 9576-9584.

Yasumoto, E., K. Nakano and T. Nakayachi. 2004. Cytotoxic activity of deferiprone, maltol and related hydroxyketones against human tumor cell lines. Anticancer Res. 24(2B): 755.

Zhang, Q. H., F. Schlyter and G. Birgersson. 2000. Bark volatiles from nonhost angiosperm trees of spruce bark beetle, Ips typographus (Coleoptera: Scolytidae): Chemical and electrophysiological analysis. Chemoecology. 10(2): 69-80.

Zhao, S. P. and Z. Xue. 1992. Studies on the chemical constituents of Cornus officinalis Sieb et Zucc. Acta Pharm. Sin. 27(11): 845.

Zhu, Y. F., Z. G. Liu and H. Liu. 2018. Fast-pyrolysis compounds of Dalbergia odorifera based on PY-GC-MS analysis. Acta Agric. Jiangxi. 30(4): 83-87. 